\title{
Finite Element Modelling of a Hearing Contact Lens Coupled to the Middle Ear
}

Dmitrii Burovikhin, Reutlingen Research Insitute, Reutlingen University, Alteburgstr. 150, 72762 Reutlingen, Germany, dmitrii.burovikhin@reutlingen-university.de

Michael Lauxmann, Reutlingen Research Insitute, Reutlingen University, Alteburgstr. 150, 72762 Reutlingen, Germany, michael.lauxmann@reutlingen-university.de

\section{Introduction}

The hearing contact lens (HCL) is an innovative type of hearing aid devices that uses a piezo-electric actuator to drive the ossicular chain at the tympanic membrane. Compared to conventional hearing aid devices, the HCL is capable of amplifying higher frequencies, which is generally important for spatial hearing and the understanding of speech. Besides, it is practically invisible to the outside observer. The goal of this study is to investigate how well the HCL can amplify the higher frequencies compared to acoustic excitation and to optimize its performance with the help of a finite element (FE) model.

\section{Methods}

The middle ear and the HCL were modelled in ANSYS Workbench. Both models as well as the combined model were validated through vibrational measurements conducted on the HCL and temporal bones using a Laser-Doppler-Vibrometer. The transfer behavior between the input voltage and the stapes motion was simulated to evaluate HCL performance, which was optimized by varing geometrical and material properties of the individual HCL components. The properties of the contact area between the HCL and the tympanic membrane were also investigated to achieve a better fit to the measurements.

\section{Results}

The simulated frequency response of the combined FE model fits well to the measurements. The transfer function was optimized to achieve a better amplification factor in the higher hearing frequency range compared to acoustic excitation. In addition, some design changes are proposed to increase the HCL performance.

\section{Conclusion}

The physical behavior of a human middle ear coupled with the HCL is modelled using FE approach. The model helped to optimize the overall performance of the HCL. Moreover, such problems as the feedback, which causes whistling sounds in the ear, the sound transfer loss in higher frequencies, the poor sound quality, and the occlusion efect can be now investigated with its help. 


\title{
3D Printed Individualized Frontal Neo-Ostium Implant in Endoscopic Sinus Surgery - a Proof of Concept Study
}

\author{
Ziwen Gao ${ }^{1,3 *}$, Farnaz Matin ${ }^{1}$, Chunjiang Wei ${ }^{1,3}$, Thomas Lenarz ${ }^{1,3}$, Constantin Weber ${ }^{1}$, Samuel John ${ }^{2}$, Verena Scheper ${ }^{1,3}$ \\ ${ }^{1}$ Department of Otolaryngology Hannover Medical School, Hanover, Germany \\ ${ }^{2}$ HörSys, Hannover, Germany \\ ${ }^{3}$ Cluster of Excellence"Hearing4all" EXC 1077/1, Oldenburg/Hannover, German Research Foundation \\ *Corresponding authors: Gao.Ziwen@mh-hannover.de, Scheper.Verena@mh-hannover.de
}

\section{Introduction}

Endoscopic sinus surgery (ESS) is the standard procedure for surgery of most paranasal sinus diseases. Ongoing inflammation and restenosis may limit the theraphy effect. This study investigates the work flow of manufacturing and implantation of 3D-printed individualized neo-ostium implants.

\section{Methods}

Six cadavers of adult participants were enrolled. An experienced surgeon performed the bilateral endoscopic sinus surgery and removing tissue from the frontal ostium to created the frontal neo-ostium. Images of the area to be implanted were taken by digital volume tomography (DVT; xCAT, Xoran Technologies, Inc., USA). The frontal neo-ostium area was segmented manually using 3D Slicer ${ }^{\mathrm{TM}}$ version 4.11 (http://www.slicer.org) and the respective DICOM (Digital Imaging and Communications in Medicine)-datasets. After segmentation, the implant surface was processed by applying the surface smoothing effect with the dimensionless parameter of 0.5 in $3 \mathrm{D}$ Slicer ${ }^{\mathrm{TM}}$. All reconsructions were transformed into a hollow object with a wall thickness of $1.5 \mathrm{~mm}$ and exported as STL (standard tessellation language) file for 3D printing. The frontal neo-ostium implant model was printed using a 3D-Bioplotter (Envisiontec) and silicon which was crosslinked using UV-light. The printed sample was sterilized using UV-light for 30 minutes. The individualized implants were inserted into the corresponding neo-ostium by an experienced surgeon. In one case the blinded surgeon received the left implant for the right sinus and the right implant for the left sinus to investigate if the surgeon recognises the side-specific individuality.All surgeries were documented by video and photographs and a final DVTimage was taken. The fitting of the individualized implant was validated on those post-implantation images and the volume and surface area of the frontal neo-ostium implants were measured.

\section{Results}

The work flow of surgery - imaging - segmentation - printing - sterilization - implantation - imaging and verification of implant position worked very well. The process chain from the first image to the sterile implant ready to be inserted toke about 90 minutes. The surgeon rated the texture as suitable for insertion. The orientation of the implant during insertion was in some cases not clear for the surgeon and had to be discussed with the technical team. The insertion was very smooth in all cases exept of the one where the surgeon tried to insert the right implant in the left sinus. Here the surgeon reported massive problems and was not able to insert the implant with the air way kept open. The silicone used was stable enough to be inserted but in a few cases the implant was damaged a little bit where the surgeon hold it with the surgical instruments. The post-surgery DVTs revealed a correct position of all 12 implants and no additional trauma to no-go areas such as the eye or brain. The volume, surface area and shape of the individual implants varied videly between left and right side in one patient and between patients.

\section{Conclusion}

The work flow of manufacturing and implantation of individualized 3D-printed frontal neo-ostium implants is established using silicone as model material. The time the surergy team has to wait for the implant is too long and has to be optimized by e.g. automatization of segmentation, shortening the UV-silicone curing time or by avoiding the sterilization process by printing already in sterile conditions such as directly in the operating theatre. The implant design has to be improved to directly inform the surgeon on the correct orientation of insertion. The analysis of the implant dimensions and anatomy of the frontal neo-ostium and the position of the implant in situ reveal that individualized implants are needed to supply the patients and that our implant design allowes a safe implantation due to its individualized design. 


\section{Transoral video-laryngoscopic surgery of the larynx}

Patrick J. Schuler, Department of Otorhinolaryngology, Head and Neck Surgery, Ulm University Medical Center, Ulm, Germany, patrick.schuler@uniklinik-ulm.de

Leon Schild, Department of Otorhinolaryngology, Head and Neck Surgery, Ulm University Medical Center, Ulm, Germany, leon.schild@uni-ulm.de

Linus Kienle, Department of Otorhinolaryngology, Head and Neck Surgery, Ulm University Medical Center, Ulm, Germany, linus.kienle@uni-ulm.de

Felix Böhm, Department of Otorhinolaryngology, Head and Neck Surgery, Ulm University Medical Center, Ulm, Germany, felix.boehm@uniklinik-ulm.de

Lüder A. Kahrs, Department of Mathematical and Computational Sciences, University of Toronto, Canada, lakahrs@cs.toronto.edu

Thomas K. Hoffmann, Department of Otorhinolaryngology, Head and Neck Surgery, Ulm University Medical Center, Ulm, Germany, t.hoffmann@uniklinik-ulm.de

\section{Introduction}

In the treatment of laryngeal carcinoma transoral laser surgery is a common procedure. However, it requires extention of the cervical spine in order to generate a straight line of sight for the surgeon. This is not possible in all patients. A nonlinear system could provide better access to the larynx in patients with insufficient cervical spine reclination.

\section{Methods}

We present a prototype of a self-constructed surgical system for transoral laryngeal surgery. It consists of a video-laryngoscope (cMAC, Storz, Tuttlingen), guiding tubes, and flexible instruments (DiLumen, Lumendi). The system was tested on a user study $(n=6)$ in an animal model (porcine larynx) and on an intubation dummy for access and visualization of the larynx. In addition, surgical mock procedures were performed in a human body donor.

\section{Results}

In the animal model, the time to reach all anatomical landmarks in the larynx correlated with the experience of the participants (49s vs. 91s). The change of instruments was carried out independently by all participants, and the time to put the surgical instruments in optimal position for a mock cordectomy was $128 \mathrm{~s} \pm 32 \mathrm{~s}$. In the intubation dummy, access of the larynx was still feasible after restricting the cervical spine reclination. In the human body donor, the prototype was superior for the visualisation of the larynx as compared to a standard laryngoscope.

\section{Conclusion}

In pre-clinical tests, the surgical system offers the possibility to manipulate laryngeal structures in a non-linear manner. In the future, this may provide a cost-effective, minimally invasive alternative in case of insufficient laryngeal exposure. A clinical pilot study will be initiated based on these results. 


\section{Development of a predictive middle-ear model for the model-based di- agnosis of middle-ear pathologies}

Benjamin Sackmann, Reutlingen Research Institute, Reutlingen University, 72762 Reutlingen, Germany, benjamin.sackmann@reutlingen-university.de

Michael Lauxmann, Reutlingen Research Institute, Reutlingen University, 72762 Reutlingen, Germany, michael.lauxmann@reutlingen-university.de

\section{Introduction}

Detection of conductive hearing loss in today's audiometric tests is mostly based on visual comparison of measurements with standard curves. However, large interindividual differences in patients' ears impede a reliable diagnosis of otherwise clearly distinctive pathologies. Model-based diagnosis would allow physics-based linkage of diagnostic information on the parameter level and better exploitation of available data. However, literature data provide very sparse information and wide parameter intervals for many of the model parameters, which contradicts the development of predictive middle-ear models for diagnostic purposes.

\section{Methods}

To overcome this issue, an adaptive parameter identification algorithm is developed to fit a finite-element model of the middle ear to results of extensive temporal bone studies with stepwise and systematically preparated pathologies. Since simple mean square error functions showed unsatisfactory results and poor convergence, we developed a problem-specific cost function based on keyfeatures. Keyfeatures comprise expert knowledge such as resonances, qualitative curve characteristics, and standard ranges from measurements. The cost function is minimized using a pattern search algorithm. Keyfeatures are recursively included in the cost function and the choice of optimization variables is based on sensitivity parameters.

\section{Results}

When jointly fitted to measurements of a normal and an otosclerotic ear, the model reproduces well the measured quantities reflectance, impedance, umbo and stapes transfer function and shows a good predictive ability of the pathologies malleus fixation and disarticulation. Furthermore, a clear assignment of identified model parameters and pathologies is achieved.

\section{Conclusion}

Sophisticated algorithms that quantitatively combine expert knowledge and measurement data enable to develop good predictive models despite sparse information on model parameters. The model parameters identified in this study highlight the importance of correct stiffness and damping middle-ear parameter ranges for correct mapping of pathological characteristics. The great sensitivity of the model with respect to pathologies shows high potential for application in model-based diagnosis. 


\title{
A Project to support Head and Neck Cancer Treatment by Artificial Intelligence - the TACTIC-HNC Trial
}

\author{
Matthaeus Stoehr ${ }^{1}$, Andreas Dietz ${ }^{1}$, Hisham Mehanna ${ }^{2}$ \\ ${ }^{1}$ Dept. of Otolaryngology, Head and Neck Surgery, University of Leipzig, Liebigstr. 12, 04103 Leipzig, Germany \\ ${ }^{2}$ Institute of Cancer and Genomic Sciences, Institute of Head and Neck Studies and Education, University of \\ Birmingham, Vincent Drive, Birmingham B15 2TT, United Kingdom
}

Email: stoehr@medizin.uni-leipzig.de

\section{Introduction}

Head and neck cancer (HNC) treatment is becoming more individualized and therefore more complex. Also, treatment may cause functional deficits and late toxicities that need to be characterized better to identify the best outcomes in terms of treatment outcomes, organ-function preservation, and quality of life. For this purpose, the application of artificial intelligence (AI) tools, particularly machine learning, for the generation of predictive models for treatment outcomes in HNC promise to improve disease prognosis and assist clinical decisions in patients.

\section{Methods}

The TACTIC-HNC trial, designed collaboratively between the Head and Neck Cancer International Group (www.hncig.org) and Savanna (an AI healthcare company) is the first ever multi-national trial on HNC management utilising AI.

It aims to describe the clinical pathway of patients with HNC in a realworld setting by analysing readily available information in Electronic Health Records. This study will gain a deep insight of the clinical characteristics and real-world outcomes of patients with all stages of HNC. It will focus on developing two predictive models to apply in the clinical setting, one for selecting patients with high-risk of recurrence after radical treatment, and the second one for selecting recurrent or metastatic patients who could benefit from immunotherapy. Advanced statistics and machine learning tools will be applied to conduct a dynamic risk stratification that will allow the evaluation of the risk of recurrence at different timepoints along the treatment pathway

\section{Results}

Cancer centres around the world are currently invited to collaborate in this trial. So far, participants from more than 10 countries worldwide are participating.

We will present the study rationale, present the study protocol and design, and explain the AI methodologies involved.

\section{Conclusion}

The TACTIC-HNC trial is inviting cancer treatment centres to collaborate in the trial to improve patients' outcomes and quality of life by support of AI. 